\title{
Same procedure as last year, James ... same procedure as every year ...
}

\author{
Jon Karlsson $^{1} \cdot$ Roland Becker $^{2} \cdot$ Michael T. Hirschmann $^{3} \cdot$ Volker Musahl $^{4}$
}

Published online: 21 November 2015

(C) European Society of Sports Traumatology, Knee Surgery, Arthroscopy (ESSKA) 2015

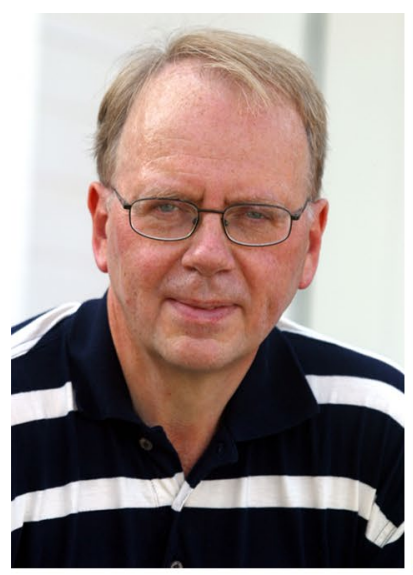

Jon Karlsson

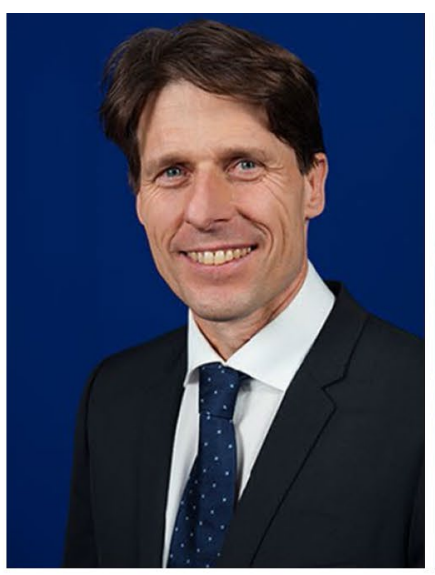

Roland Becker

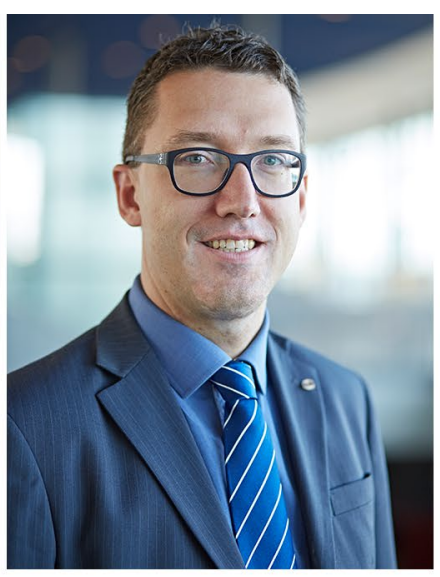

Michael T. Hirschmann

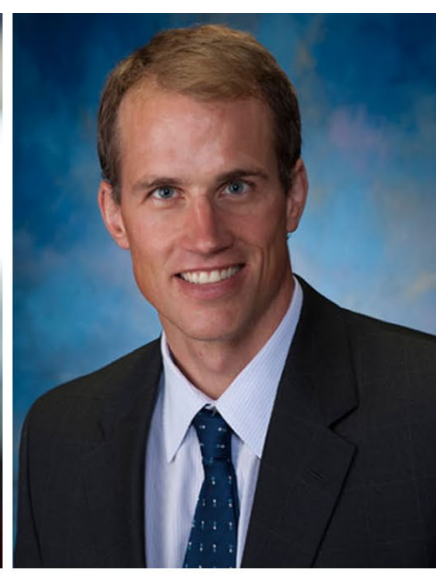

Volker Musahl

Jon Karlsson

jon.karlsson@telia.com

Roland Becker

roland.becker@kssta.org

Michael T. Hirschmann

Michael.Hirschmann@unibas.ch

Volker Musahl

musahlv@upmc.edu

1 Department of Orthopaedics, Sahlgrenska University Hospital, Mölndal, Sweden

2 Department of Orthopaedic and Traumatology, City Hospital Brandenburg, Hochstrasse 29, 14770 Brandenburg/Havel, Germany

3 Department of Orthopaedic Surgery and Traumatology, Kantonsspital Baselland (Bruderholz, Liestal, Laufen), Bruderholz, Switzerland

4 Orthopaedic Surgery and Bioengineering, University of Pittsburgh, UPMC Center for Sports Medicine, 3200 South Water Street, Pittsburgh, PA 15203, USA

Another year has come to an end, it's strange how time flies. And, it is time for the same procedure as last year, i.e. the journal has grown, submissions have increased, the Impact Factor has increased. We are happy and proud to be the journal's leadership.

Again, we would like to start by thanking all the reviewers listed below. You are extremely valuable to us, and without you, the journal would not be the same. Most probably, there would be no journal at all. We are aware of the fact that reviewing a manuscript is timeconsuming, sometimes boring and it is unpaid. A second problem is that many of you as good reviewers serve several journals. KSSTA is still an European journal, but as of today, we are more and more international. Therefore, we have increasingly tried to find new reviewers from outside Europe. We are aware that all too often the best reviewers are hit with review requests from several journals at the same time. We, therefore, try to limit the number of reviews assigned to the same reviewer as 
much as possible; a maximum of six reviews per year is the goal. During 2015, we have cleaned the reviewer list and reduced the numbers by more than half; we currently have around 500 on our list. We strongly feel that this will lead to improvements, better and more timely reviews, as we have kept only the very best reviewers. Already, we feel that the review process has improved substantially during the year.

We will as in the previous years select the best reviewers of the year, and the award will be given during the ESSKA Congress in Barcelona in May 2016. We are currently in the middle of the selection process. Once more, thank you reviewers for all your great efforts during this year. Your work is invaluable, and without you, the journal would soon be out of business.

Our website is now well consolidated (www.kssta. org) and is well coordinated with the new ESSKA website (www.esska.org), and we now have more than 500 Facebook friends. We sincerely hope that all these activities are rewarding for the authors and the readers of the journal. We continuously update information on the website and Facebook. Thanks to Sebastian Kopf, our web-editor.

Last June, we were really happy to learn that KSSTA's Impact Factor for 2014 had climbed to 3.053. We now have reached the milestone of an Impact Factor of +3 for the first time. This places the journal in the top 10 position in both categories of Sports Medicine and Orthopaedics, where we climbed to the 7th place among more than 80 journals worldwide. As mentioned many times before, improvements are still possible and we need to improve the Impact Factor even further in the future. We are happy with improvements, but we will not rest. There are many other issues that we need to improve, like the turnaround time, i.e. the time between submission and decision and the time taken for the 2 nd and 3rd reviews. And, we need to shorten the backlog; in this aspect, we have not succeeded so well. We probably need to increase the rejection rate in order to cope with the increasing number of submissions.

So, what are the facts? Is it the same procedure as lastyear, the same procedure as every year? Yes, we might say so; more of the same, but a little bit better. The journal has in fact increased and improved in every aspect. We published almost 4000 pages this year (a record number) and will do so also in 2016 (including several upcoming
Themes Issues that we are currently working on), and the number of submissions has risen to almost 1,600 (also a record number). The constantly increased flow of papers is good, but as we have decided not to expand the journal more, this will lead to more papers being rejected; some are rejected already at the Editorial level, when we (the Editors) strongly feel that the manuscript does not fit well into the journal and/or the scientific news value is limited. However, we are very happy with the trend we have noted; i.e. many high-quality papers are being published in the journal today. We know that this will lead to greater readership and an improved impact factor.

We have made some major changes to the Editorial Team during 2015. Roland Becker is now Deputy Editorin-Chief and the two new Assistant Editors-in-Chief are Michael Hirschmann and Volker Musahl. The new Assistant Editors-in-Chief have already made a huge impact and off-loaded the chief Editors in several ways, something that was much needed. We (Roland and Jon) would like to welcome Michael and Volker to the top table, and we look forward to long-term collaboration. We have also made some major changes to the Editorial Board, and we would like to welcome several new Editorial Board members. These persons have all done outstanding work as reviewers during the last years and will no doubt do so in the future too. We will continue to look for the best people for the Editorial Board. Finally, we have completely restructured the Editorial Office in Luxembourg with two new Editorial Assistants. We are very happy with their efficacy and their own ideas to bring the journal forward.

We would like to especially thank Runeeta Rai and Amanda Olsson, who run the Editorial Office, the ESSKA Board and the ESSKA Office in Luxembourg for good collaboration during the year. Thanks to Zhanna Kovalchuk, who runs the ESSKA Office. As always, our friends at Springer, especially Gabriele Schröder for outstanding work. The journal is produced in India, also an outstanding editorial team. Thanks to you all.

Finally, we would like to wish you all a Happy New Year in 2016. The journal will meet new challenges, but we feel that the Editorial team and the Editorial Office are strong and we are ready to face all the challenges ahead. We will certainly do our best. We are looking forward to seeing you all at the ESSKA Congress in Barcelona in May 2016. We will organize several interesting KSSTA activities there. Have fun! 


\section{5 reviewers}

Henrik Aagaard

Pierre Abadie

Jeffrey S. Abrams

Paul W. Ackermann

Nobuo Adachi

Eva Ageberg

Jens Agneskirchner

Mattias Ahldén

Sufian S. Ahmad

Maeyama Akira

Eduard Alentorn-Geli

Fredrik Almqvist

Tjarco D. W. Alta

Bogdan Ambrozic

Andrew A. Amis

Thor Einar Andersen

Jack Taylor Andrish

Peter Angele

Hari Krishna Ankem

Pierluigi Antinolfi

Daisuke Araki

Elizabeth A. Arendt

Markus P. Arnold

Carl Askling

O Sahap Atik

Emmanuel Audenaert

Jesper Augustsson

Olufemi R. Ayeni

Martin Bachmann

Ji-Hoon Bae

Greg Bain

Corrado Bait

Klaus Bak

Paul Baker

Peter Balcarek

Andrea Baldini

Maurice Balke

Ingo J. Banke

Adad Baranto

Björn Barenius

Kristoffer Weisskirchner Barfod

Gomez Barrena

John Bartlett

Tiberiu Bataga

Thomas Bauer

Mike H. Baums

Philippe Beaufils

Christoph Becher

Roland Becker

Johannes Beckmann

Asheesh Bedi
Henrik Behrend

Knut Beitzel

Etienne Belzile

Franco Benazzo

Massimo Berruto

Simone Bignozzi

Onur Bilge

Erkal Bilgic

Rudi Georg Bitsch

Mario Bizzini

Hanna Cecilia Björnsson Hallgren

Lars Blönd

Michael Bohnsack

Tommaso Bonanzinga

Michel P. Bonnin

Ulrich Bosch

Tim Boymans

Kristín Briem

Karen K. Briggs

Martijn Brinkman

Helena Brisby

Mats Brittberg

Robert Brophy

Peter U. Brucker

Stefan Budde

Paolo Bulgheroni

Bruce Burnham

Matteo Cadossi

James Calder

Carlo Camathias

Stefano Campi

Mike Carmont

Antonio Cartucho

Enric Castellet

Antonia F. Chen

ChengKung Cheng

Franck Chotel

Mustafa Citak

Thomas Clanton

Benjamin Clarsen

Martin Clauss

Justin Cobb

Andres Combalia

Vincenzo Condello

Norberto Confalonieri

Louis Conrad

Fabio Conteduca

Nelson Cortes

P. Maxwell Courtney

Ramon Cugat

Diane L. Dahm

Kiriakos Daniilidis

Nikica Darabos
Thomas De Bo

Ricardo de Casas

Marco De Gori

Lieven De Wilde

Koen Defoort

Masataka Deie

David Henri Dejour

Guillaume Demey

Brian Meldan Devitt

Giovani Di Giacomo

Tobias Johannes Dietrich

Patrick Djian

Simon Donell

Mahmut Nedim Doral

Klaus Dieter Draenert

Matej Drobnic

Jon Olav Drogset

Lutz Duerselen

Victoria Duthon

Irem Duzgun

Fredrik Einarsson

Lars Erik Ejerhed

Carl Ekholm

Henry Bone Ellis

Nathan Kincaid Endres

Lars Engebretsen

Mehmet Erdil

Bengt I. Eriksson

Karl Eriksson

Lucio Ernlund

Aidin Eslam Pour

João Espregueira-Mendes

Max Ettinger

Simon A. Euler

Denise Eygendaal

Jaroslaw Fabis

Filippo Familiari

Jack Farr

Brian T. Feeley

Julian Ashley Feller

Julian Fernandez-Gonzalez

Mario Ferretti

Andrea Ferretti

Matthias Jens Feucht

Christian Fialka

Giuseppe Filardo

Clare Fitzpatrick

Simon Fogerty

Fernando Fonseca

Philipp Forkel

Francisco Forriol

Magnus Forssblad

Edoardo Franceschetti 
Francesco Franceschi

Alois Franz

Niklaus F. Friederich

Michael Frink

Karl-Heinz Frosch

Freddie H. Fu

Susanne Fuchs-Winkelmann

John Fulkerson

Edward MH Gardner

Håkan Gauffin

Christian Gerhardt

Alan Getgood

Jeroen Geurts

Sandro Giannini

Thomas Gill

Francesco Giron

John none Gliatis

Alberto Gobbi

Alli Gokeler

Pedro Hinarejos Gomez

Andreas Gomoll

Jon Goosen

Karin Grävare Silbernagel

Hans Granhed

Alberto Grassi

Nicolas Graveleau

Stefan Grote

Daniel Guenther

Chinmay Gupte

Martin Hägglund

Jürgen Höher

Per Hölmich

Michiel Hageman

Andreas Halder

Camilla Halewood

Seung-Beom Han

Milan Handl

Michael Elias Hantes

Horia Haragus

Philippe Hardy

Arsi Harilainen

Joseph M. Hart

Nael Hawi

Petra Heesterbeek

Annette Heijne

Johann Henckel

Philipp Henle

Daniel Hensler

Mirco Herbort

Elmar Herbst

Lee Herrington

Benton Heyworth

Stefan Hinterwimmer
Michael Tobias Hirschmann

Anna Hirschmann

Alfred Hochrein

Martijn Hofman

Siegfried Hofmann

Theresa Holmgren

Yuichi Hoshino

Stephen Miller Howell

James Hui

Christophe Hulet

Ingrid Hultenheim-Klintberg

Mark Hutchinson

Andreas B. Imhoff

Eivind Inderhaug

Sheila Jean McNeill Ingham

Michael Iosifidis

Takanori Iriuchishima

James Irrgang

Yasuyuki Ishibashi

Michael Jagodzinski

Per-Mats Janarv

Rob P. A. Janssen

Timo Jarvela

Mislav Jelic

Jean-Yves Jenny

Jae-Heon Jeong

Joerg Jerosch

Urban Johnson

Jim Johnson

Brigitte Jolles-Haeberli

Peter Kälebo

Mustafa Karahan

Jon Karlsson

Jüri Kartus

Defne Kaya

Sugimori Kazuhito

John G. Kennedy

Gino Kerkhoffs

Armin Keshmiri

Oliver Kessler

Tommi Kiekara

Chlodwig Kirchhoff

Christoph Kittl

Karsten Knobloch

Gunnar Knutsen

Koichi Kobayashi

Baris Kocaoglu

Hideyuki Koga

Jason L. Koh

Sandro Kohl

Dieter Kohn

Elizaveta Kon

Sui Wah Kong
Lars Konradsen

Sebastian Kopf

Nanne Pieter Kort

Sentaro Koshida

Ioannis Kostogiannis

Ladislav Kovacic

Natascha Kraus

Michael Rindom Krogsgaard

Aaron J. Krych

Ryosuke Kuroda

Joanna Kvist

Sverre Löken

Christian Lüring

Matthias Lahner

Christoph lampert

Robert F. LaPrade

Lior Laver

Keith Lawhorn

Marie-Eve LeBel

Yeon Soo Lee

Sang Hak Lee

Dae-Hee Lee

Peter de Leeuw

Nicolas Lefevre

Alberto Lemos

Andreas Lenich

Simon Lenschow

Pisit Lertwanich

Pazit Levinger

Bruce A. Levy

Ofer Levy

Guoan Li

Sven Lichtenberg

Michael C. Liebensteiner

Dennis Liem

Albert Lin

Martin Lind

Anders Lindahl

Anders Lindstrand

Alexis Lion

Nicola Lopomo

Mattia Loppini

Olaf Lorbach

Joerg Luetzner

Mari Lundberg

Sebastien Lustig

Stephen Lyman

Jack Lysholm

C. Benjamin Ma

Peter Macdonald

Henning Madry

Nicola Maffulli

Robert A. Magnussen 
Suzanne Maher

Mahir Mahirogullari

Martin Majewski

Konstantinos G. Makridis

Laurent Malisoux

Maurillio Marcacci

Giulio Maria Marcheggiani Muccioli

Fabrizio Margheritini

Stefan Marlovits

Antonio Marmotti

Frank Martetschläger

Vladimir Martinek

Inigo Martinez

Cesar A. Q. Martins

Alessandro M. Massã

Philippe Massin

Dimitrios Mastrokalos

Tomoyuki Matsumoto

Takehiko Matsushita

Craig Mauro

Hermann-Otto Mayr

Jodie Adele McClelland

David A. McGuire

Omer Mei Dan

Khaled Meknas

G. L. Melegati

Duncan Edward Meuffels

Christophe Meyer

Yutaka Mifune

Radovan Mihelic

Giuseppe Milano

Matthew D. Milewski

Maarten H. Moen

Stefan Mogos

Juan C. Monllau

Hong-Kyo Moon

Nuno Moura

Caroline Mouton

Andreas Mueller

Thomas Muellner

Lars Muller

Takeshi Muneta

Christopher D. Murawski

Matthew Murray

David Murray

Volker Musahl

Gregory Myer

Sara Myers

Peter Myers

Truls Straume-Næsheim

Takumi Nakagawa

Norimasa Nakamura

Wolfgang Nebelung
Sven Nebelung

Manfred Nelitz

Valentin Neuhaus

Aaron Biing Ng

Philipp Niemeyer

Kjell Nilsson

Katarina Nilsson-Helander

John Nyland

Mitsuo Ochi

Shawn O'Driscoll

Joo Han Oh

Francesco Oliva

Nicklas Olsson

Patrick Orth

Geert Pagenstert

Hemant Pandit

Géza Pap

Pericles Papadopoulos

Dietrich Pape

Evangelos Pappas

Min Jong Park

Sebastien Parratte

Nikolaos K. Paschos

Rahul Patel

Thilo Patzer

Jochen Paul

Stephan Pauly

Christopher J. Pearce

Luigi Adriano Pederzini

Hélder Miguel Duarte Pereira

Giuseppe Peretti

Frank A. Petrigliano

Martin Pietsch

Anand Pillai

Kevin Plancher

Boris Poberaj

Guiseppe Porcellini

Andrew Porteous

Chadwick C. Prodromos

Thomas Pufe

Nicolas Pujol

Jan Harald Rötterud

Hans Rahme

Pietro Randelli

Filippo Randelli

Eva Rasmussen Barr

Bjoern Rath

Mikel L. Reilingh

Per Renstrã

Stavros Ristanis

Scott Rodeo

Frank Roemer

Harald Roos
Roberto Rossi

Claudio Rosso

Miguel Angel Ruiz Iban

Arcangelo Russo

Octav Russu

Junnosuke Ryu

Anne Kathrine Belling Sörensen

Greg Saboeiro

Patrick Sadoghi

Mo Saffarini

Björn Salomonsson

José Filipe Magro e Silva Salreta

Massimiliano Salvi

Gian M. Salzmann

Gonzalo Samitier

Kristian Samuelsson

Mikel Sanchez

Vicente Sanchis-Alfonso

Mikael Sansone

Dominique Saragaglia

Martin Sauerschnig

Sven Scheffler

Markus Scheibel

Alfredo Schiavone Panni

Oliver Schindler

Phillip Schottle

Roberto Seijas

Romain Seil

Ninni Sernert

Elvire Servien

Ellen Shanley

Fran Sheehan

Seth Sherman

Antony Shield

Eiji Shimpuku

Konsei Shino

Sebastian Siebenlist

Rainer Siebold

Petri Sillanpaa

Luminita Simion

Einar Andreas Sivertsen

Inge Skråmm

Toby O. Smith

Jay Smith

Francesc Soler

Eirik Johan Solheim

Gunter Spahn

Tim Spalding

Kurt P. Spindler

Sander Spruijt

Christian Stärke

Sven Stener

Nicholas Stergiou 
Karl Stoffel

Robin Kinnear Strachan

Sjoerd Stufkens

Jorma Styf

Stefan Patrick Sussmann

Daisuke Suzuki

Imre Szerb

Magnus Tägil

Goro Tajima

Norimasa Takahashi

Masato Takao

Koji Takayama

Reha Tandogan

Daniel Theisen

Kathi Thiele

Emmanuel Thienpont

Chuck Thipgen

Roland Thomee

Kristian Thorborg

Fritz Thorey

Jonas Bloch Thorlund

Thomas Tischer

Marc Tompkins

Michael Torry

Roy Tranberg

Elias Tsepis

Gabrielle J. M. Tuijthof

Efe Turgay
Nikolaos Emmanouil Tzanakakis

Srikant Vallabhajosula

Ewould Van Arkel

Christiaan van Bergen

Sebastiaan van de Groes

Michel P. J. van den Bekerom

Olivier A. J. van der Meijden

Willem Mare Van der Merwe

Nick C. van Dijk

Carola F. van Eck

Nicolien Van Giffen

Ronald Johannes van Heerwaarden

Corné J. M. van Loon

Alexander Van Tongel

Remco van Wensen

Pieter-Jan Vandekerckhove

Hilde Vandenneucker

Haris S. Vasiliadis

Michele Vasso

Patrick Vavken

Jordi Vega

Alberto Ventura

Rene Verdonk

Nico Verdonschot

Bruno Violante

Piero Volpi

Daniel Wagner

Christopher Wahl
Ola Wahlström

Bernhard Waibl

Markus Waldén

William Robert Walsh

Sandeep Wasnik

Patrick Weber

Lars Weidenhielm

Andre Weimann

Danny Whelan

Harald Widhalm

Wojciech Widuchowski

Lotta Willberg

Jaap Willems

Philippe Guy Claude Wilmes

Ivan H. Wong

Diederick Bernard Wouters

Alexis Wright

Kazunori Yasuda

Yon-Sik Yoo

Shinichi Yoshiya

Alastair Younger

Stefano Zaffagnini

Franceska Zampeli

Eva Zeisig

Boris Zelle

Qiuxia Zhang 